\title{
CD36 Genotype and Long-Chain Fatty Acid Uptake in the Heart
}

\author{
Taigo Kintaka, MD; Takao Tanaka, MD; Makoto Imai, MD; \\ Itaru Adachi, MD*; Isamu Narabayashi, MD*; Yasushi Kitaura, MD
}

\begin{abstract}
Homozygous or compound heterozygous mutation of the $C D 36$ gene $\left(\mathrm{CD}^{-1-}\right)$ in humans results in severe defects of the uptake of long-chain fatty acids (LCFAs) in the heart. Because the effect of a single mutation of this gene $\left(\mathrm{CD} 36^{+/-}\right)$on the LCFA uptake is not known, it was evaluated in 29 subjects with the CD36 wild-type gene (WT) (6 healthy subjects, 10 patients with heart disease), $\mathrm{CD}^{+/-}$(4 healthy subjects, 5 patients) and $\mathrm{CD} 36^{-/}$ (4 patients). The CD36 genotype was identified in the coding region of genomic DNA, and the expression of CD36 protein was examined by flow cytometry after staining with monoclonal anti-CD36 antibody. The LCFA uptake in the heart was assessed as the radioactivity accumulation ratio of heart to mediastinum after intravenous administration of iodine-123 15-( $p$-iodophenyl)-3- $R, S$-methylpentadecanoic acid (H/M ratio). The H/M ratios in WT, CD36 $6^{+-}$and CD36- $6^{--}$were $2.28 \pm 0.10,1.90 \pm 0.06$ and $1.40 \pm 0.11$, respectively ( $<<0.0001$, among groups). The H/M ratio between healthy subjects and patients with heart disease for WT and $\mathrm{CD} 36^{+/}$did not differ significantly (ie, those of WT and CD36 ${ }^{+-}$in healthy subjects and patients were $2.29 \pm 0.08$ vs $2.27 \pm 0.12$ and $1.90 \pm 0.07$ vs $1.89 \pm 0.05$, respectively). Not only $\mathrm{CD} 36^{-/-}$but also $\mathrm{CD} 36^{+/-}$resulted in a significant reduction of the LCFA uptake in the heart independent of heart disease, suggesting genotype dependency and that CD36 might be a fundamental determinant of myocardial LCFA uptake. (Circ J 2002; 66: 819-825)
\end{abstract}

Key Words: CD36; Genotype; Long-chain fatty acid uptake; Myocardium; Scintigraphy

A though the heart preferentially uses long-chain fatty acids (LCFAs) as its main energy substrate, alteration in LCFA utilization is well known in pathological hearts ${ }^{1}$ and in such cases, assessment of the LCFA metabolism is of clinical importance in determining etiology and developing therapeutic strategies.

LCFA metabolism in the heart has been clinically evaluated by scintigraphy using iodine-123-15-( $p$-iodophenyl)3- $R, S$-methylpentadecanoic acid (BMIPP), a radioactive LCFA analog. Regional defects of myocardial LCFA uptake are often revealed in patients with coronary artery disease ${ }^{2-5}$ and have been interpreted as follows: (1) dissociation between BMIPP and flow-tracer activity (ie, reduced BMIPP uptake but less reduced flow-tracer accumulation) may indicate ischemic but still viable myocardium (ie, the 'mismatch of blood flow and metabolism'); or (2) diminished accumulation of BMIPP together with reduced flowtracer radioactivity related to necrotic or fibrotic tissue.

However, despite the increase of flow tracer accumulation in the heart, defects of BMIPP uptake have been noticed in patients with hypertrophic cardiomyopathy and interestingly, an almost negative depiction of the heart by BMIPP scintigraphy has been occasionally observed in patients without discernible defects of coronary perfusion.-8 The underlying mechanism of this discrepancy (ie, decreased uptake of LCFA in the heart despite rather increased uptake of flow-tracer) has not been fully elucidated.

(Received April 17, 2002; revised manuscript received June 4, 2002; accepted June 10, 2002)

Third Division, Department of Internal Medicine and *Department of Radiology, Osaka Medical College, Osaka, Japan

Mailing address: Takao Tanaka, MD, PhD, Third Division, Department of Internal Medicine, Osaka Medical College, Takatsuki, Osaka 569-8686, Japan. E-mail: in3024@ poh.osaka-med.ac.jp
The first step of cellular LCFA metabolism is a transverse process of LCFA in the plasma membrane. Although this process is still in dispute, several lines of evidence suggest a protein-mediated process in addition to a simple diffusive process. Among the putative proteins involved,

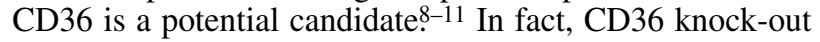
mice have severe defects of LCFA uptake in the heart ${ }^{10}$ and homozygous and compound heterozygous mutation of the CD36 gene (hereafter referred to as $\mathrm{CD}^{-1-}$ ) in humans also causes severe defects of myocardial LCFA uptake, with an almost negative depiction of the heart by BMIPP scintigraphy?

In addition to the effects of CD36-- on the LCFA uptake in the heart, $\mathrm{CD} 36^{-/-}$resulted in a lack of CD36 expression in platelets and monocytes, referred to as type I CD36 deficiency 12 We recently found a strong association between the genotype in the coding region of $C D 36$ and the expression level of CD36. Heterozygous mutations in the coding region of this gene (hereafter referred to as $\mathrm{CD} 36^{+/-}$) resulted in the reduced expression of CD36 in monocytes, approximately half that observed in the wild-type gene (hereafter referred to as WT) $!^{13}$ Accordingly, we hypothesized that not only $\mathrm{CD} 36^{-/-}$but also $\mathrm{CD} 36^{+/-}$might lead to the defects of LCFA uptake in the heart.

The effects of CD36-1- on the LCFA uptake in the heart have been characterized, so far, only qualitatively; that is, an almost negative depiction of the heart by BMIPP scintigraphy. The quantitative evaluation of LCFA uptake in the heart has been analyzed in a few studies using the radioactivity accumulation ratio of heart to mediastinum $(\mathrm{H} / \mathrm{M})$ after intravenous administration of BMIPP ${ }^{14,15}$ Knowing the mutational effects on the LCFA uptake in the heart should further clarify the role of CD36. In this study, we investigated the quantitative LCFA uptake in the heart 
Table 1 Characteristics of Subjects

\begin{tabular}{|c|c|c|c|c|c|c|c|c|}
\hline $\begin{array}{l}\text { Case } \\
\text { no. }\end{array}$ & $\begin{array}{c}\text { Age } \\
\text { (years) }\end{array}$ & Sex & $\begin{array}{l}\text { Cardiac } \\
\text { condition }\end{array}$ & & & Monocytes & Platelets & $H / M$ \\
\hline$D 1$ & 44 & $F$ & $H C M$ & CD $36^{-/-}$ & $C 478 T+$ Ins 1159A & 0.00 & 0.4 & 1.24 \\
\hline$D 2$ & 46 & $M$ & $P V C$ & $C D 36^{--}$ & Homo (C478T) & 0.01 & 0.2 & 1.44 \\
\hline D3 & 67 & $M$ & $D C M$ & $C D 36^{-/-}$ & $C 478 T+D e l 539 A C$ & 0.01 & 0.06 & 1.45 \\
\hline D4 & 34 & $M$ & HCM & $C D 36^{-/-}$ & $C 478 T+D e l 539 A C$ & 0.00 & 0.1 & 1.48 \\
\hline$S P 1$ & 64 & $M$ & $H C M$ & $C D 36^{+/-}$ & $C 478 T$ & 16.5 & 13.2 & 1.85 \\
\hline$S P 2$ & 39 & $F$ & $D C M$ & $C D 36^{+/}$ & $C 478 T$ & 12.4 & 15.7 & 1.86 \\
\hline$S P 3$ & 65 & $M$ & $H T$ & $C D 36^{+/}$ & $C 478 T$ & 19.0 & 20.5 & 1.88 \\
\hline †SP4 & 61 & $F$ & $V S A$ & $C D 36^{+/}$ & $C 478 T$ & 11.4 & 0.3 & 1.88 \\
\hline SP5 & 39 & $F$ & $H C M$ & $\mathrm{CD} 36^{+/-}$ & $C 478 T$ & 17.3 & 15.0 & 1.97 \\
\hline SN1 & 27 & $M$ & Norm. & $C D 36^{+/-}$ & $C 478 T$ & 13.1 & 10.3 & 1.83 \\
\hline$S N 2$ & 36 & $M$ & Norm. & $C D 36^{+/}$ & Del539AC & 11.5 & 15.5 & 1.86 \\
\hline SN3 & 39 & $M$ & Norm. & $C D 36^{+/-}$ & C478T & 13.7 & 12.8 & 1.95 \\
\hline SN4 & 35 & $M$ & Norm. & $C D 36^{+/-}$ & $C 478 T$ & 18.0 & 11.4 & 1.96 \\
\hline$W P 1$ & 55 & $M$ & $H C M$ & $W T$ & None & 30.7 & 24.9 & 2.18 \\
\hline$W P 2$ & 64 & $F$ & $H C M$ & $W T$ & None & 31.6 & 15.6 & 2.18 \\
\hline WP3 & 64 & $M$ & $V S A$ & $W T$ & None & 24.7 & 18.1 & 2.18 \\
\hline WP4 & 59 & $M$ & $H T$ & $W T$ & None & 22.1 & 11.0 & 2.25 \\
\hline WP5 & 61 & $M$ & $H T$ & $W T$ & None & 32.1 & 15.2 & 2.30 \\
\hline WP6 & 75 & $M$ & $H T$ & $W T$ & None & 45.9 & 37.1 & 2.39 \\
\hline WP7 & 78 & $M$ & $C A D$ & $W T$ & None & 30.5 & 26.1 & 2.51 \\
\hline WP8 & 52 & $F$ & $H C M$ & $W T$ & None & 22.3 & 26.4 & 2.35 \\
\hline WN1 & 35 & $M$ & Norm. & $W T$ & None & 33.3 & 20.6 & 2.17 \\
\hline$W N 2$ & 59 & $M$ & Norm. & $W T$ & None & 39.6 & 13.6 & 2.24 \\
\hline WN3 & 37 & $M$ & Norm. & $W T$ & None & 32.3 & 25.8 & 2.28 \\
\hline WN4 & 28 & $M$ & Norm. & $W T$ & None & 34.8 & 20.0 & 2.31 \\
\hline WN5 & 51 & $M$ & Norm. & $W T$ & None & 40.7 & 28.8 & 2.35 \\
\hline WN6 & 43 & $M$ & Norm. & $W T$ & None & 23.2 & 6.5 & 2.41 \\
\hline tWP9 & 53 & $F$ & $D C M$ & $W T$ & None & 25.4 & 0.1 & 2.13 \\
\hline WP10 & 72 & $M$ & VSA & $W T$ & None & 27.9 & 0.4 & 2.25 \\
\hline
\end{tabular}

Abbreviations: Charact., characteristic; Norm., healthy volunteer; PVC, premature ventricular contraction; HCM, hypertrophic cardiomyopathy; DCM, dilated cardiomyopathy; VSA, vasospastic angina; HT, hypertension; CAD, coronary artery disease; CD36--, homozygous or compound heterozygous mutation of the CD36 gene; CD36+-, heterozygous mutation; WT, wild-type gene; None, no mutation was detected in the coding region of the FAT/CD36 gene; Homo (C478T), homozygous mutation of C478T; C478T+Ins1159A, CD36-- for C478T and Ins1159A; C478T+Del539AC, CD36-/ for C478T and Del539AC; H/M, heart to mediastinum radioactivity ratio; ${ }^{\dagger}$, no expression of $C D 36$ protein in platelets but expression in monocytes.

using BMIPP in subjects with WT, CD36+/- and CD36--. WT and $\mathrm{CD} 36^{+/-}$groups included both patients with heart disease and healthy subjects.

\section{Methods}

\section{Study Population}

The study population consisted of 29 unrelated Japanese subjects (10 healthy male volunteers; age range, 27-59 years and 19 patients with heart disease 12 males, 7 females; age range, 39-72 years) (Table 1 ).

WT, $\mathrm{CD} 36^{+/-}$and $\mathrm{CD} 36^{-/-}$were found by flow cytometry with fluorescein isothiocyanate (FITC)-labeled monoclonal antibody (FA6-152, Marseille, France) and the expression of CD36 protein was assessed as mean fluorescent intensity (MFI)!3 Mutations were identified by genomic analysis in the CD36 coding region of genomic DNA? Volunteers were free of any disease, had an unremarkable medical history and were not taking any medication at the time of the investigation. The diagnoses of the patients were based on medical history, physical examination, electrocardiogram, chest radiography, echocardiography, and coronary angiography. Patients were treated at outpatients clinics and were not severely ill (New York Heart Association class I-II).

The investigation conformed to the principles outlined in the Declaration of Helsinki, and written informed consent was obtained from all the subjects.

\section{BMIPP Myocardial Scintigraphy and the Heart-to-} Mediastinum Radioactivity Ratio (H/M ratio)

Both planar and single photon emission computed tomography (SPECT) imaging of all the subjects were done with a standard acquisition method. After overnight fasting, subjects had an intravenous injection of $111 \mathrm{MBq}$ of iodine-123-15-( $p$-iodophenyl)-3- $R, S$-methylpentadecanoic acid (I-123 BMIPP; Nycomad-Amersham, UK) at rest. A three-head SPECT system (GCA-9300A, Toshiba Medical System, Tokyo, Japan) equipped with a low-energy high-resolution collimator was used to acquire anterior planar images with a $512 \times 512$ matrix for $5 \mathrm{~min}$ at $30 \mathrm{~min}$ after injection. Next, SPECT projection data with a $64 \times 64$ matrix were obtained from a 360-degree circular orbit in 60 steps ( 30 s per step) with a $20 \%$ window centered on the $160 \mathrm{keV}$ photopeak. The projection data were processed using a GMS-550 WorkStation (Toshiba Medical System). The reconstruction was performed by the filtered back projection technique with a Butterworth filter. Reconstruction slices were set parallel to the short, vertical long, and horizontal long axes.

Radioactivity was counted in the region of interest (ROI), which was manually drawn over the whole heart by a nuclear-cardiologist (I.A.) unaware of the CD36 genotype, from the planar image (Fig 1). To evaluate regional 
(A)

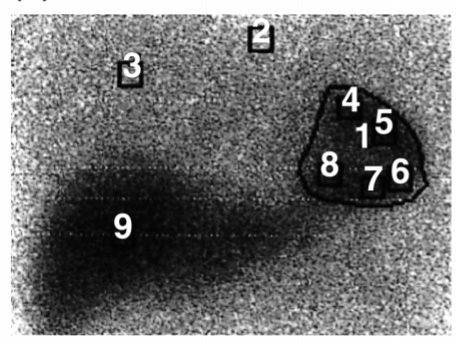

(B)

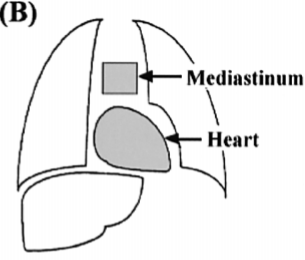

(C)

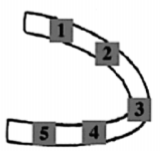

Fig 1. Measurements of BMIPP radioactivity in the whole-heart region, the contour of which was hand-defined, and in the mediastinum region (A), as schematically illustrated in (B). Regional myocardial radioactivity was measured in 5 regions of the heart, as schematically illustrated in $(\mathrm{C})$.
(A)

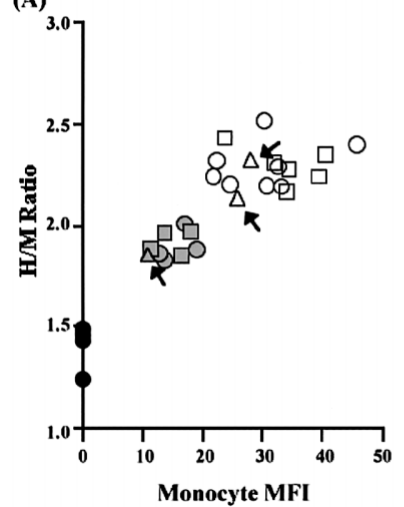

(B)

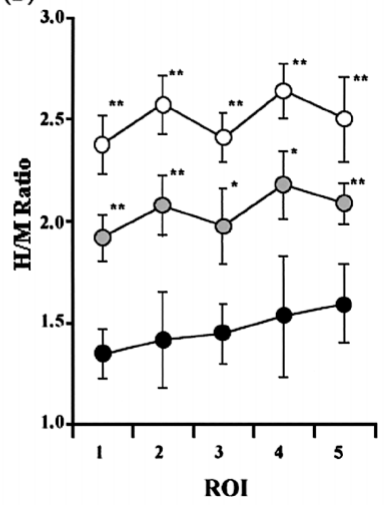

Fig 3. Whole-heart H/M ratio and monocyte MFI (A), and regional $\mathrm{H} / \mathrm{M}$ ratio (B). (A) Relation between the whole-heart $\mathrm{H} / \mathrm{M}$ ratio (vertical axis) and monocyte MFI (horizontal axis) in each individual. The filled, hatched and open symbols represent subjects with homozygous or compound heterozygous mutation of the CD36 gene $\left(\mathrm{CD} 36^{-1-}\right)$, heterozygous mutation $\left(\mathrm{CD} 36^{+--}\right.$) and wild-type gene (WT), respectively. Squares and circles represent healthy subjects and patients with heart disease, respectively. Subjects lacking CD36 protein expression in platelets but present in monocytes (type II CD36 deficiency) are indicated as open and hatched triangles with arrows. (B) The H/M ratio in the 5 regions of the heart. Closed, hatched, and open circles represent subjects with $\mathrm{CD} 36^{-1-}, \mathrm{CD} 36^{+-}$and WT, respectively. ${ }^{*} \mathrm{p}<0.0001$ in $\mathrm{CD} 36^{-/-}$vs $\mathrm{CD} 36^{+/-}$and $\mathrm{CD} 36^{+/-}$vs WT, respectively; $* \mathrm{p}<0.005$ in $\mathrm{CD} 36^{-/-}$vs $\mathrm{CD} 36^{+/}$.

LCFA uptake, 5 ROIs ( $3 \times 3$ pixels in size) were placed on the myocardial image obtained from the planar image (Fig 1). The ROI was also placed over the mediastinum and used as the background activity. The H/M ratio was calculated as a fraction of the mean counts per pixel in the heart divided by those in the mediastinum.

\section{Statistical Analysis}

Data are presented as mean \pm standard deviation (SD). The data were compared by one way analysis of variance (ANOVA) with the Scheffe's test. A value of $\mathrm{p}<0.05$ was considered significant.

\section{Results}

Characteristics of the Subjects (Table 1)

There were 16 subjects with the WT: 6 healthy subjects

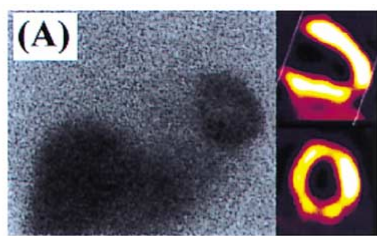

Wild-type

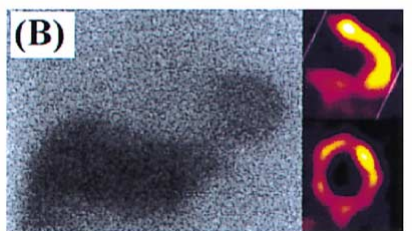

Heterozygote (C478T)

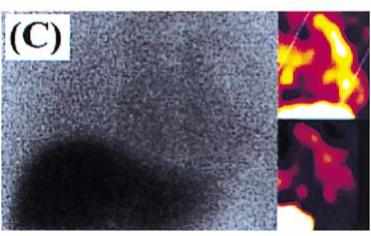

Homozygote (C478T)
Fig 2. Representative scintigrams in subjects with the wild-type CD36 gene, heterozygous mutation of C478T, and homozygous mutation of $\mathrm{C} 478 \mathrm{~T}$.
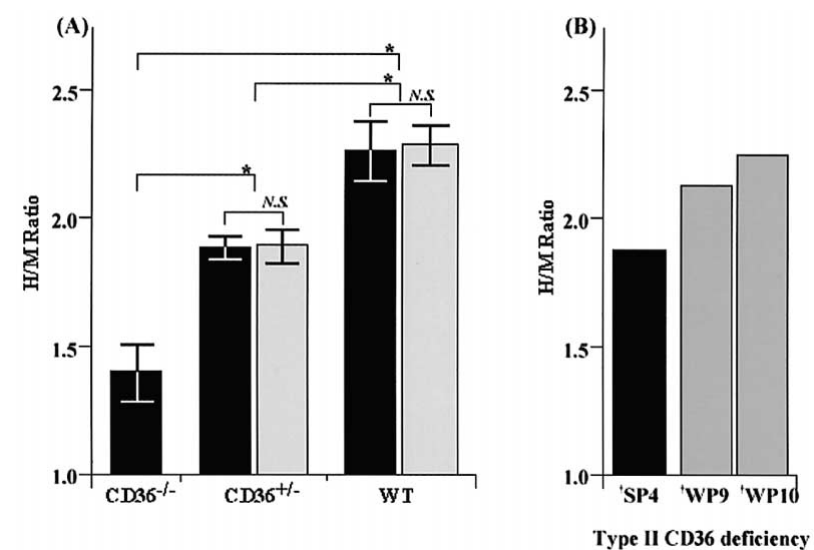

Fig 4. The H/M ratio in healthy subjects and patients with heart disease (A) and in patients with type II CD36 deficiency (B). (A) Whole-heart $\mathrm{H} / \mathrm{M}$ ratio in patients with heart disease and in healthy subjects with CD36 $6^{-1}$, CD $36^{+-}$and WT. Closed and shaded bars represent patients with heart disease and healthy subjects, respectively. Patients with $\mathrm{CD}^{-1-}: n=4$; patients with $\mathrm{CD}^{-1} 6^{+-}: n=5$; healthy subjects with $\mathrm{CD}^{+1-}: n=4$; patients with WT: $n=8$; healthy subjects with WT: $n=6$. ${ }^{*} \mathrm{p}<0.0001$ among groups, NS, not significant. (B) Whole-heart H/M ratio in patients with type II CD36 deficiency. The closed bar is SP4 in Table 1 and the shaded bars are WP9 and WP10, respectively, in Table 1. SP4 was CD36+- and WP9 and WP10 were WT.

and 10 patients with heart disease, including vasospastic angina, hypertension, coronary artery disease, dilated cardiomyopathy and hypertrophic cardiomyopathy. There were 9 subjects with $\mathrm{CD}^{+/-}$: 8 for $\mathrm{C} 478 \mathrm{~T}$ and 1 for Del539AC; 4 were healthy subjects and 5 were patients, including vasospastic angina, hypertension, dilated cardiomyopathy and hypertrophic cardiomyopathy. There were 4 patients with $\mathrm{CD}^{-1-}$ : 1 homozygous mutation $\left(\mathrm{CD} 36^{-1-}\right)$ for $\mathrm{C} 478 \mathrm{~T}, 1$ compound heterozygous mutation $\left(\mathrm{CD} 36^{-/-}\right)$ for $\mathrm{C} 478 \mathrm{~T}$ and Ins1159A, and $2 \mathrm{CD}^{-1-}$ for $\mathrm{C} 478 \mathrm{~T}$ and Del539AC.

\section{H/M Ratio}

Representative SPECTs from subjects with WT, CD36 ${ }^{+/-}$ and $\mathrm{CD}^{-1-}$ are shown in Fig 2. The relation between the whole-heart $\mathrm{H} / \mathrm{M}$ ratio (vertical axis) and monocyte MFI (horizontal axis) is shown in Fig 3A. The whole-heart H/M 
Table 2 Mutations, H/M Ratio and Expression of CD36 Protein in Monocytes and Platelets

\begin{tabular}{lcccc}
\hline \hline Mutation & $n$ & $\begin{array}{c}\text { H/M ratio } \\
(\text { mean } \pm S D)\end{array}$ & $\begin{array}{c}\text { Monocyte MFI } \\
(\text { mean } \pm S D)\end{array}$ & $\begin{array}{c}\text { Platelet MFI } \\
(\text { mean } \pm S D)\end{array}$ \\
\hline$C D 36^{-/}$ & 4 & $1.40 \pm 0.11$ & $0.01 \pm 0.01$ & $0.19 \pm 0.15$ \\
$C D 36^{+-}$ & 9 & $1.90 \pm 0.06^{*}$ & $14.8 \pm 2.94^{*}$ & $12.7 \pm 5.53^{*}$ \\
$W T$ & 16 & $2.28 \pm 0.10^{*}$ & $31.7 \pm 6.47^{*}$ & $18.1 \pm 10.3^{N S}$ \\
\hline
\end{tabular}

CD36--, homozygous or compound heterozygous mutation of the CD36 gene; CD36+--, heterozygous mutation; WT, wild-type gene; H/M ratio, whole-heart to mediastinum radioactivity ratio; MFI, mean florescence intensity; SD, standard deviation.

${ }^{*} p<0.0001 \mathrm{CD}^{*} 6^{-/-}$vs CD36 $6^{+/}$, and CD36+/- vs WT, respectively. NS, No significant difference between CD36 $6^{+/}$and WT $(p=0.16)$.

ratio representing LCFA uptake in the heart showed a good correlation with monocyte MFI representing expression level of $\mathrm{CD} 36$ in monocytes. The whole-heart $\mathrm{H} / \mathrm{M}$ ratios in subjects with WT, CD $36^{+/-}$and $\mathrm{CD}^{-1-}$ were $2.28 \pm 0.10$, $1.90 \pm 0.06$, and $1.40 \pm 0.11 \quad(\mathrm{p}<0.0001$ among groups $)$ (Table 2). Although the $\mathrm{H} / \mathrm{M}$ ratios in the basal, midventricular and apical regions demonstrated a significant and homogeneous reduction in subjects with $\mathrm{CD}_{3} 6^{+/}$and $\mathrm{CD}^{2} 6^{-1-}$ compared with the WT, those the respective genotypes stayed within the same range of magnitude (Fig 3B). The whole-heart $\mathrm{H} / \mathrm{M}$ ratio between healthy subjects and patients with heart disease did not significantly differ in either WT $(2.29 \pm 0.08$ in healthy subjects and $2.27 \pm 0.12$ in patients) or $\mathrm{CD}^{2} 6^{+/-}(1.90 \pm 0.07$ in healthy subjects and $1.89 \pm 0.05$ in patients) (Fig 4 ).

\section{H/M Ratio and Expression of CD36 Protein}

The H/M ratio in each individual was strongly associated with the monocyte MFI (Fig 3A), but not with platelet MFI in either WT or CD36+l- . Platelet MFI in both WT and $\mathrm{CD}^{2} 6^{+-}$was distributed in a broad range (0.1-37.1 in WT and 0.3-20.5 in CD36 ${ }^{+/}$, Table 1) and no significant difference of platelet MFI was observed between the 2 genotypes ( $\mathrm{p}=0.16$, Table 2$)$. However, the $\mathrm{H} / \mathrm{M}$ ratio in both $\mathrm{WT}$ and $\mathrm{CD}^{+/-}$subjects was within the same range of magnitude (2.17-2.51 in WT and 1.83-1.97 in CD36 ${ }^{+-}$, Table 1) and significant difference of the $\mathrm{H} / \mathrm{M}$ ratio was observed between them $(\mathrm{p}<0.0001$, Table 2$)$. Consequently, we speculated that the LCFA uptake in the heart and the expression of CD36 in monocytes were regulated by the same or closely related factor(s), but was not the case for the expression of CD36 in platelets.

A phenotype lacking CD36 expression in platelets, but present in monocytes, has been reported and is referred to as type II CD36 deficiency! ${ }^{16}$ We had 3 cases in the present study (SP4, WP9 and WP10 in Table 1) and we investigated their CD36 genotype, H/M ratio, and expression of CD36 in platelets and monocytes.

Case SP4 was CD36+/- for C478T, but the other 2 cases (WP9 and WP10) did not demonstrate any mutation in the coding region of $C D 36$. The $\mathrm{H} / \mathrm{M}$ ratio in SP4 was 1.88 and 2.13 and 2.25, respectively, for WP9 and WP10 (Fig 4). The monocyte MFI from Case SP4 was 19.0, and 25.4 and 27.9 for Cases WP9 and WP10, respectively. This finding also suggests that the LCFA uptake in the heart and the expression of CD36 in monocytes, but not in platelets, strongly depend on the CD36 genotype.

\section{Discussion}

We studied the effects of mutations of the $C D 36$ gene on the LCFA uptake in the heart and demonstrated the following.
(1) Mutations of this gene, not only CD36 $6^{-/-}$but also $\mathrm{CD}^{2} 6^{+/-}$, resulted in a significant reduction of the LCFA uptake in the heart compared with the WT.

(2) The uptake of LCFA in the heart was strongly associated with the $C D 36$ genotype independent of heart disease.

(3) The type II CD36-deficient phenotype is heterogeneous and did not always accompany a defect in myocardial LCFA uptake.

\section{LCFA Uptake in the Heart and CD36 Genotype}

The cardiac image on the BMIPP scintigram was almost invisible in patients with $\mathrm{CD}_{36^{-1-}}$, but was clearly visible for those subjects with either $\mathrm{CD} 36^{-/-}$or WT and did not differ qualitatively between them (Fig 2). However, the $\mathrm{H} / \mathrm{M}$ ratio, a semi-quantitative assessment of the BMIPP uptake in the heart, revealed significant differences among the genotypes. The H/M ratio was significantly decreased in $\mathrm{CD}^{-6^{--}}(1.40 ; 61.4 \%$ of WT) and CD36+- $(1.90 ; 83.3 \%$ of WT) compared with WT (2.28) (Table2). CD36-lresulted in a null level of CD36 expression in monocytes and platelets and an almost negative depiction of the heart on BMIPP scintigraphy? Accordingly, we expected complete loss of myocardial radioactivity accumulation in such patients, but contrary to this expectation, substantial radioactivity was observed in their hearts, consistent with the myocardial BMIPP radioactivity observed in CD36 knock-out mice (50-80\% of control) 10 Hence, the myocardial radioactivity observed in both patients and mice implies additional myocardial LCFA uptake processes (eg, simple diffusion and/or other protein-mediated process) ${ }^{17-20}$ Assuming that the difference between the H/M ratio in a given genotype and that in $\mathrm{CD}^{-1-}$ represents the fraction of CD36-mediated LCFA uptake in the heart, those fractions in WT, CD36 $6^{+/-}$and $\mathrm{CD}^{-1-}$ were $0.88,0.5$ and 0 , respectively, suggesting a gene-dosage-dependent effect of CD36 on LCFA uptake in the heart. As well, the expression of CD36 in monocytes suggests a gene-dosage-dependency; that is, the monocyte MFI in WT, CD $36^{+/-}$and $\mathrm{CD}^{-1-}$ was $31.7 \pm 6.47,14.8 \pm 2.94$ and 0 , respectively (Table 2). We could not analyze CD36 expression in cardiomyocytes, but we consider it likely that functional CD36 protein is not expressed in cardiomyocytes of the $\mathrm{CD} 36^{-/-}$ genotype and are expressed at half the level of the WT in the $\mathrm{CD} 36^{+/-}$subjects, which may account for the differences in myocardial BMIPP uptake among the 3 genotypes.

\section{LCFA Uptake in the Heart and Heart Disease}

Modifications of LCFA utilization in the heart are well known in pathological hearts ${ }^{1}$ and the reduced myocardial radioactivity observed in the present study thus might be a consequence of disease-induced pathophysiology. We could not evaluate LCFA uptake in any healthy subject with $\mathrm{CD}^{2} 6^{-/}$, but were able to do so in 4 with $\mathrm{CD}^{2} 6^{+-}$. 
The H/M ratio between healthy subjects and patients with heart disease in both the WT and CD $36^{+/-}$genotypes were as follows.

(1) The whole-heart H/M ratio in both $\mathrm{CD} 36^{+/-}$and WT genotypes did not differ significantly between healthy subjects and patients with heart disease (Fig 4).

(2) The whole-heart H/M ratio in WT was comparable to the reported values in normal subjects! 14,15

(3) The reduction of myocardial BMIPP uptake in $\mathrm{CD}^{-1-}(61.4 \%$ of WT) was consistent with that observed in CD36 knock-out mice (50-80\% of control)!

(4) The whole-heart H/M ratio was rather strongly associated with the CD36 genotype independent of heart disease; that is, WT $>\mathrm{CD} 36^{+/-}>\mathrm{CD} 36^{-1-}$.

Overall, we consider that the defects of BMIPP uptake in the heart are unlikely to be a secondary consequence of heart disease. However, the number of patients in this study was small and none were severely ill (New York Heart Association class I-II). Further studies in a sufficient number of patients with a broad range of severity of heart disease are still necessary.

\section{LCFA Uptake in the Heart and Expression of CD36 Protein}

The present study included 3 patients with type II CD36 deficiency, which is a lack of CD36 in platelets but not in monocytes. The expression of CD36 in the monocytes of 2 patients (Cases WP9 and WP10) was comparable to that in the WT, but was halved in Case SP4. We identified CD36+/for C478T in Case SP4, but did not detect any mutation in Cases WP9 and WP10. The whole-heart H/M ratio and the expression of CD36 in the monocytes of Cases WP9 and WP10 were comparable to those in WT, and those in Case SP4 were comparable to those in CD36+/-.

We recently found that type II CD36 deficiency has 2 subgroups! ${ }^{13}$

(1) Platelet-restricted CD36 expression disorder(s) with the WT coding region of $C D 36$. In this group, CD36 expression is lacking in platelets, but the expression in monocytes is comparable to the WT (eg, Cases WP9 and WP10).

(2) Complex disorders; that is, platelet-restricted CD36 expression disorder(s) together with $\mathrm{CD} 36^{+/-}$in the coding region of $C D 36$. In this group, $\mathrm{CD} 36$ expression is also lacking in platelets, but in monocytes the expression is comparable to $\mathrm{CD} 36^{+/-}$, at approximately half that of the WT (eg, Case SP4).

Despite the broad range of platelet MFI in subjects with either WT or $\mathrm{CD}^{+/-}$, the H/M ratio stayed in a narrow range of magnitude in both groups. Furthermore, the H/M ratio in patients with type II CD36 deficiency was associated with the genotype in the coding region of $C D 36$ (Fig 4). Taking all the reuslts together, we consider that the genotype in the coding region of $C D 36$ strongly affects the LCFA uptake in the heart and the expression of CD36 in monocytes, but not in platelets. However, further studies in a large number of subjects will be necessary to clarify this.

\section{LCFA Analog Uptake in the Heart and Factors Influencing Radioactive Uptake}

In the present study, the radioactivity in the heart was measured at $30 \mathrm{~min}$ after intravenous administration of BMIPP. This time point was regarded as representing the LCFA uptake in the heart, because plasma radioactivity during this time period decreased sharply and then increased slowly thereafter probably because of metabo- lites of BMIPP21 However, several factors still modify the LCFA uptake in the heart.

Among them, plasma concentrations of energy substrates such as glucose and LCFA are known to affect the myocardial extraction of radioactive LCFA analogs 22 In the present study, the concentrations of plasma glucose, insulin, and free fatty acid under fasting conditions and the results of a 75-g oral glucose tolerance test did not significantly differ among the subjects with WT, CD36+/- and CD36-1- (data not shown). Thus, we consider plasma substrate concentrations had little effect on the myocardial BMIPP uptake, but further studies in large mass populations will be necessary to verify this.

Coronary artery disease is also known to cause defects of the BMIPP uptake in the heart? ${ }^{2-5}$ Myocardial perfusion assessed by thallium-201 scintigraphy did not show any detectable perfusion disorder in the 4 patients with $\mathrm{CD} 36^{-/-}$, in agreement with previous findings8 In addition, the $\mathrm{H} / \mathrm{M}$ ratio in the 5 regions of the heart decreased homogeneously and significantly in both subjects with $\mathrm{CD} 36^{--}$ and CD $36^{+/-}$compared with those with WT. Therefore, a total and/or regional disorder of coronary blood supply is unlikely to be the cause of the whole and homogeneous reduction of the BMIPP uptake in the heart.

\section{Study Limitations}

We did not include healthy subjects with CD36-1-, thus we could not compare the $\mathrm{H} / \mathrm{M}$ ratio between healthy subjects and patients with heart disease for $\mathrm{CD} 36^{-/}$. However, this flaw may be overcome by the finding of a significant reduction of myocardial BMIPP uptake in CD36 knock-out mice!10 The numbers of subjects with $\mathrm{CD} 36^{-/-}$ and $\mathrm{CD} 36^{+/-}$was relatively small and the effects of these mutations on cardiac morphology and physiology were not fully studied. Thus, we cannot suggest the pathological relevance of this gene mutation.

However, defects of myocardial BMIPP uptake with an increased accumulation of flow-tracer have been observed in $80 \%$ of patients with hypertrophic cardiomyopathy? Whether this phenomenon is a primary or secondary consequence of hypertrophic cardiomyopathy is still unclear. We have observed both $\mathrm{CD} 36^{-/-}$and $\mathrm{CD} 36^{+/-}$in patients with hypertrophic cardiomyopathy and hypothesize that there is a possible link of this gene mutation with cardiomyopathy?23,24 In contrast, Nakamura et al observed little influence of CD36 deficiency on the pathophysiology of hypertrophic cardiomyopathy; however, they did not characterize the CD36 genotype.5 The type I and II CD36-deficient phenotypes do not always show mutations in the coding region of $C D 36$ on qualitative flow cytometric analysis, ${ }^{13}$ so phenotype typing by this method is likely to overlook subjects with CD $36^{+/-}$because they show a positive expression of CD36 in both platelets and monocytes. Furthermore, in the present study subjects with type II CD36 deficiency were heterogeneous for LCFA uptake in the heart and expression of CD36 in monocytes. Thus, the inconsistency between our finding and that of Nakamura et al may, at least in part, be explained by the difference in the definition of CD36 deficiency; in other words, classification by genotype or phenotype. We believe that the characterization of the genotype in the coding region of CD36 is of fundamental importance for exploring a possible involvement of CD36 deficiency in heart disease. However, the significance of this deficiency in cardiomyopathy is the subject of further investigation. 
CD36 deficiency has also been found in spontaneously hypertensive rats ${ }^{26}$ and $\mathrm{CD} 36$ knockout mice generated by gene-knockout technology ${ }^{7}$ Observations in CD36 deficient humans and rodents have suggested the possible pathological involvement of CD36 deficiency in cardiomyopathy $8,23,24,28-30$ and altered lipid and carbohydrate handling 31,32 in humans, and spontaneous hypertension, hypertriglyceridemia, abdominal obesity, insulin resistance, $26,33,34$ cardiac hypertrophy 35 and dyslipidemia ${ }^{27}$ in rodents.

Subjects with CD $36^{+/-}$are relatively common in the Japanese population (approximately $10 \%)^{13}$ and there were 4 healthy subjects without evident cardiac manifestation in the present study. This may challenge the aforementioned possible pathological involvement and our hypothesis, but the LCFA uptake in the heart, assessed by the H/M ratio, was significantly decreased in all 4 . Hajri et al recently demonstrated the elimination of cardiac hypertrophy by dietary supplementation with short-chain fatty acids in CD36-deficient rats, 35 which suggests possible involvement of dietary and/or environmental factors on the development and/or progression of cardiac hypertrophy in individuals with mutations for the $C D 36$ gene, and may, at least in part, explain the difference in cardiac phenotype in these individuals. In addition, because of the existence of a residual LCFA uptake observed in CD $36^{-1-}$, cardiac complications might be finally develop after the cumulative repetition of disadvantageous conditions (eg, prolonged starvation, exercise or serious infection).

In summary, the uptake of LCFA in the heart was strongly associated with the CD36 genotype, independent of heart disease, suggesting a gene-dosage-dependent effect. Together with our previous report? the findings presented here strongly support our hypothesis that the $C D 36$ gene is a primary determinant of LCFA uptake in the human heart.

\section{Acknowledgments}

The authors thank Dr van der Vusse (Cardiovascular Research Institute Maastrichit, Department of Physiology, Maastrichit University) and Dr. Ryotaro Yoshida (Department of Physiology, Osaka Medical College) for their critical reading and comments on the manuscript.

\section{References}

1. Sack MN, Rader TA, Park S, Bastin J, McCune SA, Kelly DP. Fatty acid oxidation enzyme gene expression is downregulated in the failing heart. Circulation 1996; 94: 2837-2842.

2. Franken P, Dendale P, De Geeter F, Demoor D, Bossuyt A, Block P. Prediction of functional outcome after acute myocardial infarction using BMIPP and Sestamibi scintigraphy. J Nucl Med 1996; 37: $718-722$.

3. Knapp FJ, Franken P, Kropp J. Cardiac SPECT with iodine-123 labeled fatty acids: Evaluation of myocardial viability with BMIPP. $J$ Nucl Med 1995; 36: 1022-1030.

4. Nishimura T, Nishimura S, Kajiya T, Sugihara H, Kitahara K, Imai $\mathrm{K}$, et al. Prediction of functional recovery and prognosis in patients with acute myocardial infarction by ${ }^{123}$ I-BMIPP and ${ }^{201} \mathrm{Tl}$ myocardial single photon emission computed tomography: A multicenter trial. Ann Nucl Med 1998; 12: 237-248.

5. Tamaki N, Kawamoto M, Yonekura Y, Fujibayashi Y, Takahashi N, Konishi J, et al. Regional metabolic abnormality in relation to perfusion and wall motion in patients with myocardial infarction: Assessment with emission tomography using an iodinated branched fatty acid analog. J Nucl Med 1992; 33: 659-667.

6. Nishimura T. Beta-Methyl-p-(123I)-iodophenyl pentadecanoic acid single-photon emission computed tomography in cardiomyopathy. Int J Cardiol Imaging 1999; 15: 45-48.

7. Inoue $\mathrm{F}$, Hashimoto $\mathrm{T}$, Nishida $\mathrm{Y}$, Dohi $\mathrm{K}$, Matsushima $\mathrm{A}$, Sakakibara H, et al. Absence of myocardial ${ }^{123}$ I-BMIPP uptake in the presence of a normal coronary angiogram and normokinetics on a left ventriculogram. Jpn Circ J 1997; 61: 263-267.

8. Tanaka T, Nakata T, Oka T, Ogawa T, Okamoto F, Kusaka Y, et al.
Defect in human myocardial long-chain fatty acid uptake is caused by FAT/CD36 mutations. J Lipid Res 2001; 42: 751-789.

9. Abumurad N, El-Maghrabi M, Amri E-Z, Lopez E, Grimaldi P. Cloning of a rat adipocyte membrane protein implicated in binding or transport of long-chain fatty acids that is induced during preadipocyte differentiation. J Biol Chem 1993; 268: 17665-17668.

10. Coburn CT, Knapp FF Jr, Febbraio M, Beets AL, Silverstein RL, Abumrad NA. Defective uptake and utilization of long chain fatty acids in muscle and adipose tissues of CD36 knockout mice. J Biol Chem 2000; 275: 32523-32529.

11. Tanaka T, Kawamura K. Isolation of myocardial membrane longchain fatty acid-binding protein: Homology with a rat membrane protein implicated in the binding or transport of long-chain fatty acids. J Mol Cell Cardiol 1995; 27: 1613-1622.

12. Kashiwagi H, Tomiyama Y, Honda S, Kosugi S, Shiraga M, Nagao $\mathrm{N}$, et al. Molecular basis of CD36 deficiency: Evidence that a ${ }^{478} \mathrm{C} \rightarrow$ $\mathrm{T}$ substitution (proline $90 \rightarrow$ serine) in CD36 cDNA accounts for CD36 deficiency. J Clin Invest 1995; 95: 1040-1046.

13. Imai M, Tanaka T, Kintaka T, Ikemoto T, Shimizu A, Kitaura Y. Genomic heterogeneity of type II CD36 deficiency. Clin Chim Acta 2002; 321: 97-106.

14. Takeuchi T, Ido A, Kashiwagi Y, Ohi S, Hasebe N, Yamashita H, et al. Systemic and regional myocardial distribution of ${ }^{123}$ I-BMIPP in normal subjects. Kaku Igaku 1995; 32: 675-681.

15. Yoshinaga K, Tahara M, Torii H, Akimoto M, Kihara K, Tei C. Myocardial scintigraphy using iodine-12315-(p-iodophenyl)-3-R, Smethylpentadecanoic acid predicts the response to $\beta$-blocker therapy in patients with dilated cardiomyopathy but does not reflect therapeutic effect. J Cardiol 2000; 35: 343-351.

16. Kashiwagi H, Tomiyama Y, Kosugi S, Shiraga M, Lipsky RH, Nagao N, et al. Family studies of type II CD36 deficient subjects: Linkage of a CD36 allele to a platelet-specific mRNA expression defect(s) causing type II CD36 deficiency. Thromb Haemost 1995; 74: $758-763$.

17. Fujii S, Kawaguchi H, Yasuda H. Isolation and partial characterization of an amphilic 56-kDa fatty acid binding protein from rat renal basolateral membrane. J Biochem (Tokyo) 1987; 101: 679-684.

18. Trigatti B, Mangroo D, Gerber G. Photoaffinity labeling and fatty acid permeation in 3T3-L1 adipocytes. J Biol Chem 1991; 266: 22621-22625.

19. Schaffer J, Rodish H. Expression cloning and characterization of a novel adipocyte long chain fatty acid transport protein. Cell 1994; 79: $427-436$.

20. Sorrentino D, Stump D, Potter B, Robinson R, White R, Kiang C, et al. Oleate uptake by cardiac myocytes is carrier mediated and involves a $40-\mathrm{kD}$ plasma membrane fatty acid binding protein similar to that in liver, adipose tissue, and gut. J Clin Invest 1988; 82: 928-935.

21. Caveliers V, De Geeter F, Pansar I, Dendale P, Bossuyt A, Franken PR. Effect of exercise induced hyperlactatemia on the biodistribution and metabolism of iodine-123-15(p-iodophenyl)-3-R,S-methyl pentadecanoic acid in normal volunteers. Eur J Nucl Med 2000; 27: $33-40$.

22. De Geeter F, Caveliers V, Pansar I, Bossuyt A, Franken PR. Effect of oral glucose loading on the biodistribution of BMIPP in normal volunteers. J Nucl Med 1998; 39: 1850-1856.

23. Okamoto F, Tanaka T, Sohmiya K, Kawamura K. CD36 abnormality and impaired myocardial long-chain fatty acid uptake in patients with hypertrophic cardiomyopathy. Jpn Circ J 1998; 62: 499-504.

24. Tanaka T, Sohmiya K, Kawamura K. Is CD36 deficiency an etiology of hereditary hypertrophic cardiomyopathy? J Mol Cell Cardiol 1997; 29: $121-127$.

25. Nakamura T, Sugihara H, Inaba T, Kinoshita N, Adachi Y, Hirasaki S, et al. CD36 deficiency has little influence on the pathophysiology of hypertrophic cardiomyopathy. J Mol Cell Cardiol 1999; 31: $1253-1259$.

26. Aitman TJ, Glazier AM, Wallace CA, Cooper LD, Norsworthy PJ, Wahid FN, et al. Identification of Cd36 (Fat) as an insulin-resistance gene causing defective fatty acid and glucose metabolism in hypertensive rats. Nat Genet 1999; 21: 76-83.

27. Febbraio M, Abumrad N, Hajjar D, Sharma K, Cheng W, Pearce S, et al. A null mutation in murine CD36 reveals an important role in fatty acid and lipoprotein metabolism. J Biol Chem 1999; 274: $19055-19062$

28. Watanabe K, Ohta Y, Toba K, Ogawa Y, Hanawa H, Hirokawa Y, et al. Myocardial CD36 expression and fatty acid accumulation in patients with type I and II CD36 deficiency. Ann Nucl Med 1998; 12: $261-266$.

29. Nakata T, Nakahara N, Sohmiya K, Okamoto F, Tanaka T, Kawamura K, et al. Scintigraphic evidence for a specific long-chain 
fatty acid transporting system deficit and the genetic background in a patient with hypertrophic cardiomyopathy. Jpn Circ J 1999; 63: 319-322.

30. Hirooka K, Yasumura Y, Ishida Y, Komamura K, Hanatani A, Nakatani S, et al. Improvement in cardiac function and free fatty acid metabolism in a case of dilated cardiomyopathy with CD36 deficiency. Jpn Circ J 2000; 64: 731-735.

31. Miyaoka K, Kuwasako T, Hirano K, Nozaki S, Yamashita S, Matsuzawa Y. CD36 deficiency associated with insulin resistance. Lancet 2001; 357: 686-687.

32. Yanai H, Chiba H, Fujiwara H, Morimoto M, Takahashi Y, Hui S, et al. Metabolic changes in human CD36 deficiency displayed by glucose loading. Thromb Haemost 2001; 86: 995-999.
33. Pravenec M, Landa V, Zidek V, Musilova A, Kren V, Kazdova L, et al. Transgenic rescue of defective Cd36 ameliorates insulin resistance in spontaneously hypertensive rats. Nat Genet 2001; 27: 156158.

34. Pravenec M, Zidek V, Simakova M, Kren V, Krenova D, Horky K, Jachymova M, et al. Genetics of Cd36 and the clustering of multiple cardiovascular risk factors in spontaneous hypertension. J Clin Invest 1999; 103: $1651-1657$.

35. Hajri T, Ibrahimi A, Coburn CT, Knapp FFJ, Kurtz T, Pravenec M, et al. Defective fatty acid uptake in the spontaneously hypertensive rat is a primary determinant of altered glucose metabolism, hyperinsulinemia and myocardial hypertrophy. J Biol Chem 2001; 276: $23661-23666$ 